\title{
Convergence of MANET in Communication among Smart Devices in IoT
}

\author{
Tanweer Alam ${ }^{\mathrm{a}}$, Baha Rababah ${ }^{\mathrm{b}}$ \\ ${ }^{a}$ Department of Computer Science, Islamic University of Madinah, Saudi Arabia Email: tanweer03@iu.edu.sa \\ ${ }^{b}$ Department of Computer Science, University of Manitoba, Canada, Email: baha@cs.umanitoba.ca
}

Received: 02 February 2019; Accepted: 20 February 2019; Published: 08 March 2019

\begin{abstract}
In the next generation network, the physical things will enable to exchange the information among them. Internet of Things (IoT) is an emerging technology that provides facility to connect physical things with the digital world and able to exchange the information. Mobile ad-hoc networks (MANET) is consistently selfdesigning, framework less system of smart devices associated with each other remotely. Every smart device is enabled to change their locations using the mobility feature of MANET. These devices are also able to act as a bridge to exchange information between devices. MANET in the IoT becomes more attractive with its important approach in the communication among smart objects because MANET has a special feature that can create a network by own self or can connect with another huge network. In this research, the authors propose a solution that describes the convergence of MANET in the IoT. The results found in this paper have been tested and implemented using different seniors.
\end{abstract}

Index Terms: MANET, IoT, Wireless Communications, Smart Objects, Information.

(C) 2019 Published by MECS Publisher. Selection and/or peer review under responsibility of the Research Association of Modern Education and Computer Science

\section{Introduction}

Nowadays, IoT is an emerging technology and it is growing rapidly. It is exploring in every area of human life. The IoT provide facilities to identify and communicate the physical object (Smart devices) [1]. The Smart devices can transfer data in MANET across all active devices without the need for a centralized approach [2]. The sensor network is a backbone of IoT. The smart device within MANET under the IoT environment works as a router. They can exchange information among them [3]. Wi-Fi devices have more capability to send data

* Corresponding author. Tel.:

E-mail address: tanweer03@iu.edu.sa,baha@cs.umanitoba.ca 
for long distance faster than Bluetooth devices [4]. Although Wi-Fi is broadly utilized for connecting the mobile devices and providing higher speeds with longer distances coverage than Bluetooth. The development of MANET via Wi-Fi on smartphones is very rare $[5,6]$. The proliferation of wireless portable smart devices as parts of everyday life, such as PDA, mobile phones, and laptops are leading to the possibility of ad-hoc wireless communication [7]. With these types of smart devices, there is a fundamental ability to share information [8]. The basic architecture of MANET is represented in figure 1.

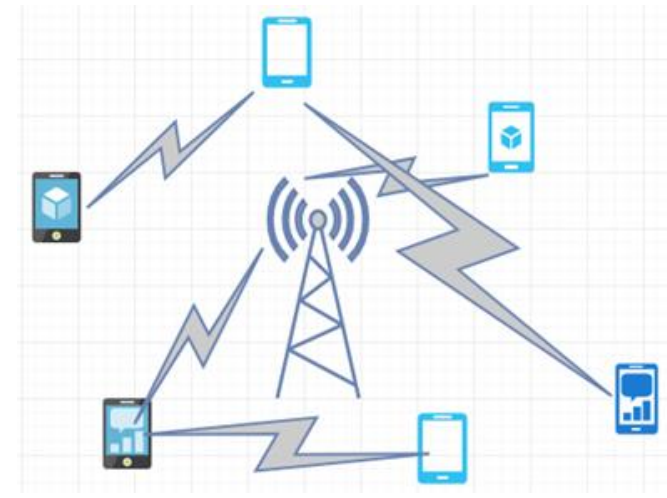

Fig.1. MANET of smart devices

In a structure-free environment, smart devices choose neighborhood through constructing expectation over accessible information to create a way of interest for the following message to be sent to the objective. Without structure approaches utilize a great deal less correspondence, the inclination can be created easily because of the versatility of the smart devices $[9,10,11]$. But there is a lot of challenges to create this kind of network. Some of them are summarised here. The objective's area should be occasionally overhauled in the structure, which presents a huge correspondence overhead. Maintaining a structure is immoderate as far as correspondence/vitality is not adaptable in MANETs setting. The rest of this paper is organized as follows 1 . Introduction, 2. Related Work, 3. Existing Models, 4. MANET Configuration, 5. Implementation of MANET in IoT 6. Represents the conclusion of the research and future scope of the proposed research.

\section{Related Work}

In the article [12], the authors presented the integrated approach of IoT and MANET using clustering techniques to balance the energy on the IoT devices because the IoT node works as a device and sometimes as a router in the MANET network. They focused on energy efficient consumptions in the IoT-MANET framework [12]. In the article [13], the authors proposed an algorithm and presented the results of the routing implementation in IoT-MANET framework. The results show the improvements in the lifetime of IoT-MANET framework [13]. In the article [14], the author presented the framework of MANET using Fuzzy controls to communicate in IoT network. In this paper, the author presented the MANET mobility models to evaluate the performance of MANET in the IoT network [14]. Thebiga.M and R.Suji Pramila were published an article [15] in the year of 2017. They described an analysis of the importance of routing protocols in MANET and IoT network. In the article [16], author presented a blockchain network importance in the IoT environment. They presented blockchains for providing the security and authenticity to the database stored in the form of blocks that are collected from smart devices of the IoT networks [16]. In the article [17], the authors get simulation results on real-world mobility traces to improve the performance of the network in heterogenous environment. In the article [18], the authors found key challenge in the MANET and IoT connectivity and they describe the secure routing protocols in the MANET-IoT network. 


\section{Existing Models used in MANET-IoT Network}

The following existing models are used in the convergence of MANET in the communications among smart devices in IoT network. The Probabilistic model-based tracking (PMT) is used to evaluate the tracking of smart devices in MANET-IoT network [19]. The Hidden Markov Model (HMM) is divided the convergence area into cells [20]. The gradient-based model (GM) is used to autoconfigure MANET to the external internet [21].

\subsection{PMT}

This protocol is used in MANET to track the IoT devices in the cell of network. It detects or sense the receiver device when it moves in the same cell of the network where the sender exist. The data is built in a disseminated way utilizing a weighted normal of the angle and the move likelihood. The angle results from smart device versatility, an IoT device experiencing the objective spares the objective's area and sets the inclination to be one that plunges as it gets more seasoned. In the accompanying, we first present the HMM model and the Gradient model, trailed by the formalization of PMT model. The figure 2 represents the average path length, average stretch factor and the success rate.

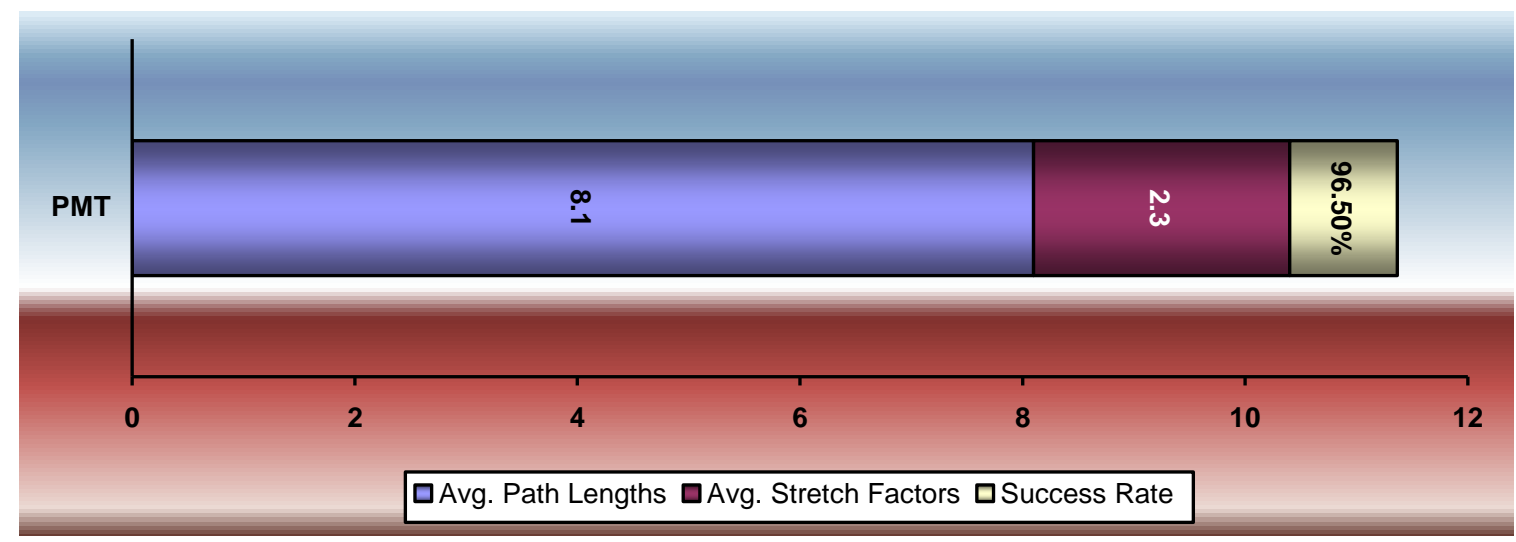

Fig.2. PMT Model statistics

\section{$3.2 H M M$}

This model is also used in tracking the targets and finding the current location of the IoT node based on the last location information that can be incorrect in some cases. It is utilized to solve the objectives of the 2D plane. The method is based on dividing the target area into cells. The transactions data in every cell is loosely synchronized to reduce coordination overhead. The figure 3 represents the comparative success rate results of PMT vs. HMM models. It shows the average path length, average stretch factor and the success rate comparisons. 


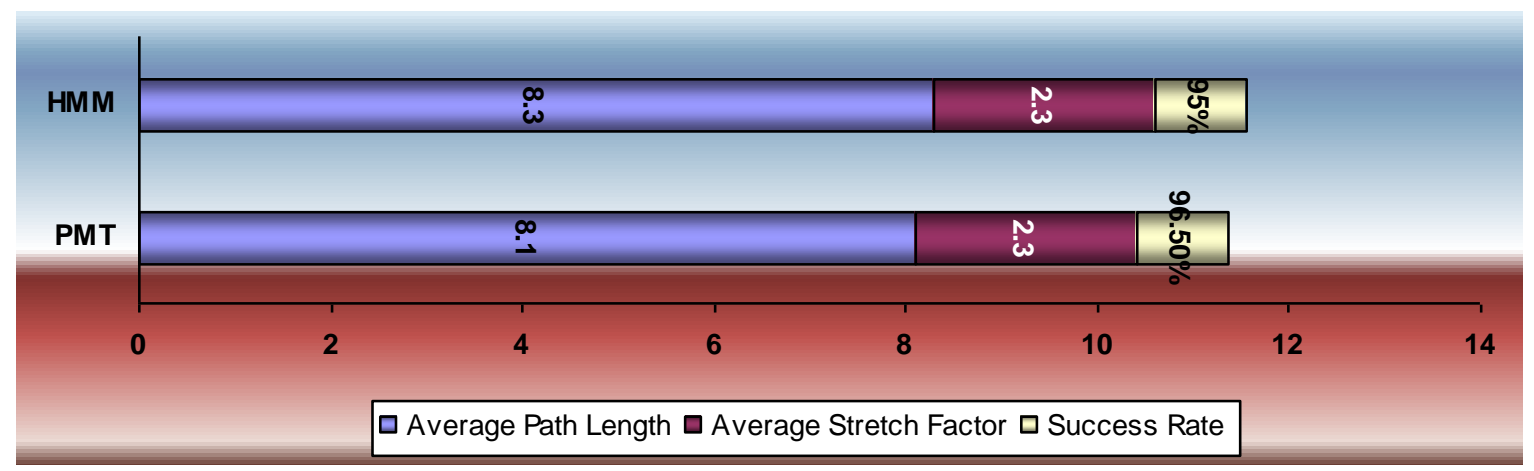

Fig.3. PMT vs. HMM Model statistics

\section{$3.3 G M$}

This model is very useful model in MANET-IoT because it performs good in the coverage of large-scale MANET in the IoT. If the IoT node track the objectives then the value of gradient will change to 1 and store its location. By this information we can track the objectives sometimes before. We have calculated the average path length, average stretch factor and the success rate and compare these results to the PMT and HMM models (figure 4).

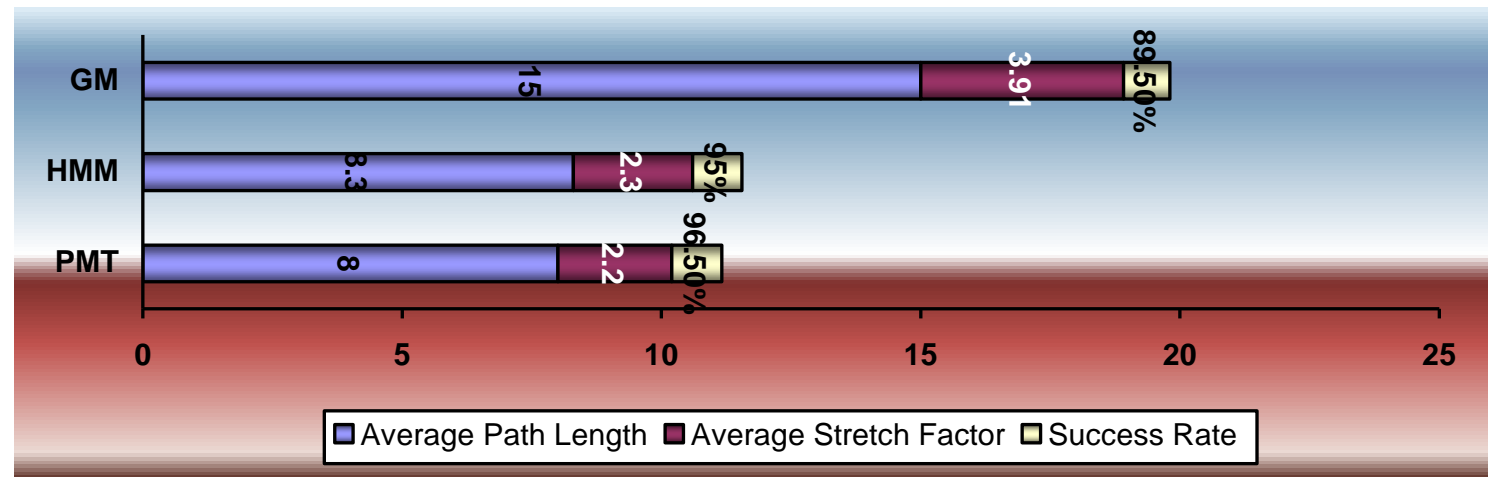

Fig.4. PMT, HMM and GM Models Comparisons

\section{MANET Configuration}

In this section, we discussed about the configuration of MANET in the IoT network. Each IoT node is expected to have a settled Wi-Fi region and an altered correspondence range. The objective is to accomplish certain reach scope and/or correspondence network prerequisites. Given an arrangement of smart devices conveyed in an objective zone, the issue is to figure out whether the region is sufficiently covered. The Coverage Configuration Protocol (CCP) that can give distinctive degrees of scope and in the interim keep up correspondence availability when the correspondence extents are no less than twice their reaches. At first, wi-fi Ad Hoc Network is in the dynamic state. In the event that a region surpasses the required level of scope, excess brilliant smart devices will get themselves pointless and switch to the rest state. A resting smart device additionally occasionally awakens and enters the listen to state. The wireless ad hoc network configuration to 
communicate with devices is implemented and utilized. The Communication between devices will be independent of the existing cellular network and will be possible whether the smart device is within range of the cellular network or not. The desired outcome of this research is to demonstrate the ability to transmit data from one device to another device using the peer-to-peer network without centralized approach.

\section{Implementation of MANET in IoT}

The MANET in IoT is gathering of autonomous portable IoT devices that can convey the information to one another through Wi-Fi waves. Smart devices that are in Wi-Fi range of one another can straightforwardly convey the information, whereas others required the aid of intermediate smart devices to route their packets of information. The link is created in the real time that makes the network completely dispersed and can work at wherever without the assistance of any access point. So that this property makes the network so strong.

The MANET of smart devices works as infrastructure less network. Rather, smart devices themselves frame the system and convey through a method for remote correspondences. Versatility causes successive topology changes and might break existing ways. In the world of smart devices, the message passing is a broadly utilized by several users. MANETs are robust, dynamic networks that can be rapidly deployed and reconfigured, making them ideal for military applications. There is an options menu that allows for smart device connection and enabling smart device discovery as well as a browser to select files to send. Smart device names are added and removed from a "connected devices" list as each smart device enters/exits the network. The tests were performed between two PandaBoards in close proximity running. The latency tests were conducted programmatically. The Monte Carlo approach is served as an exploratory application for distributed registering with smart devices.

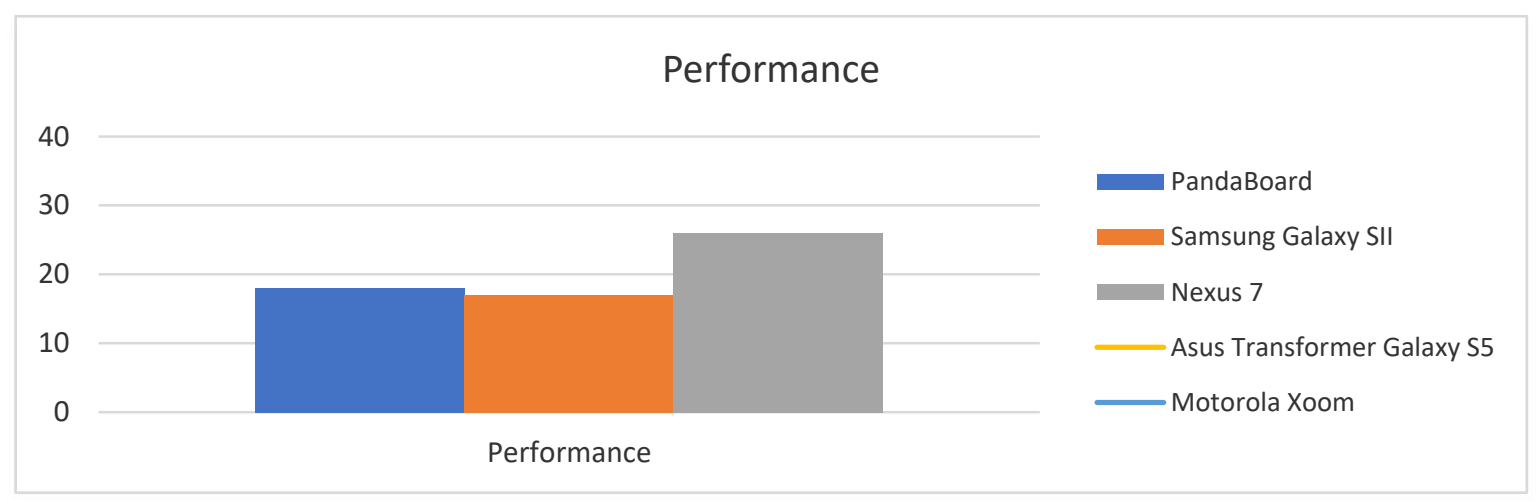

Fig.5. Single device execution times for various platforms.

The experimental test setup analysed both homogeneous and heterogeneous IoT device networks. For this exercise with block scheduling, the results obtained for uniform smart device networks outperformed the mixed device network since the workload distribution was optimal. To test, a heterogeneous network was formed by using PandaBoard, Nexus 7, Samsung Galaxy, and Asus Transformer. 


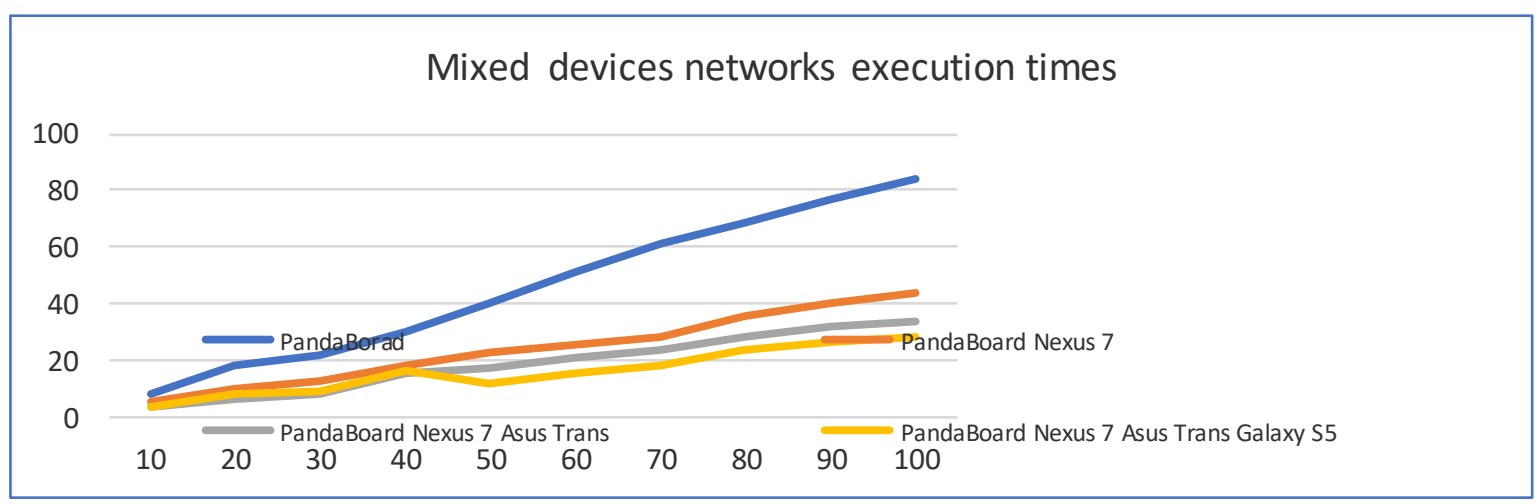

Fig.6. Mixed smart devices networks execution times.

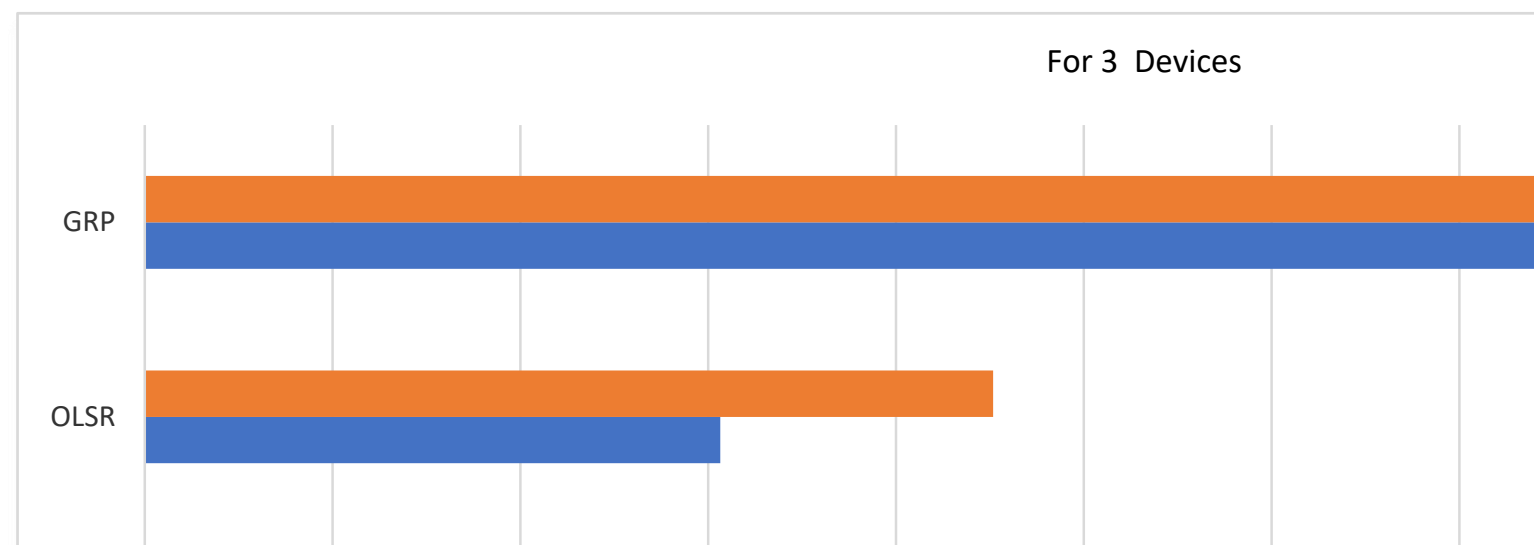

Fig.7. Throughput (3 Devices)

A graphical representation of the performance measurements achieved is presented in wi-fi. The limit and abilities of handheld smart devices keep on enhancing with handling power and the imagination of use engineers.

Table 1.omparison of AODV, OLSR, and GRP

\begin{tabular}{|c|c|c|c|c|c|c|}
\hline Throughputs & \multicolumn{2}{|c|}{ AODV-Protocol } & \multicolumn{2}{c|}{ OLSR-Protocol } & \multicolumn{2}{c|}{ GRP-Protocol } \\
\hline & Random & Vector & Random & Vector & Random & Vector \\
\hline 3 Devices & 5622 & 5612 & 3065 & 4517 & 8501 & 9234 \\
\hline 5 Devices & 2020 & 2014 & 5067 & 9087 & 22106 & 25678 \\
\hline
\end{tabular}




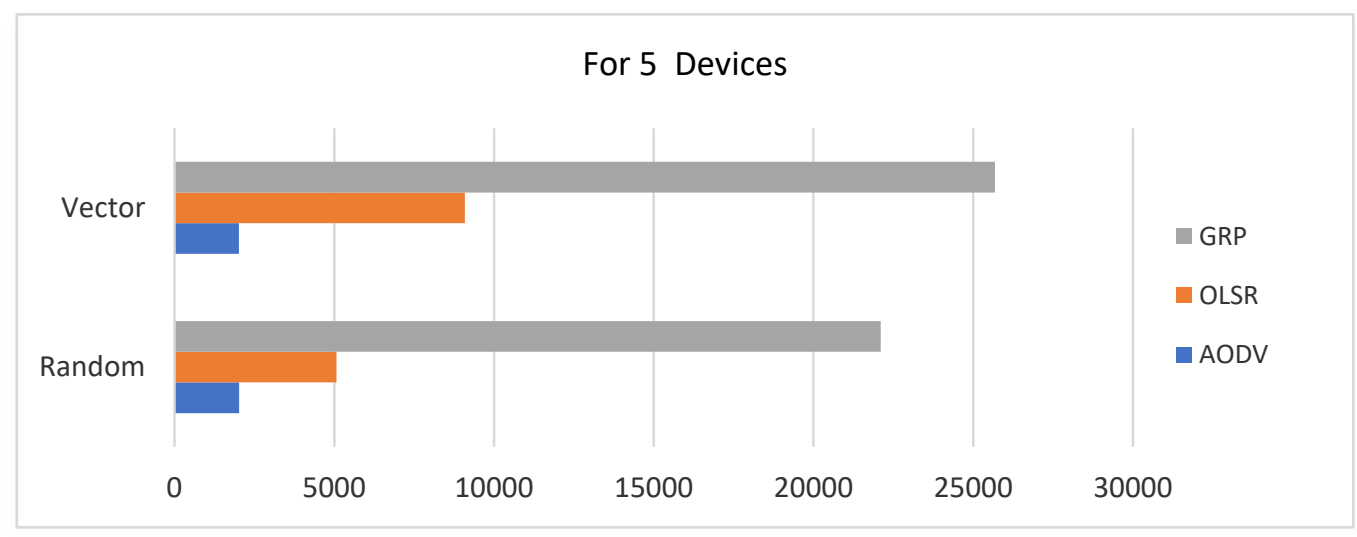

Fig.8. Throughput (5 Devices)

Delay: Delay demonstrates to what extent it a packet takes to travel.

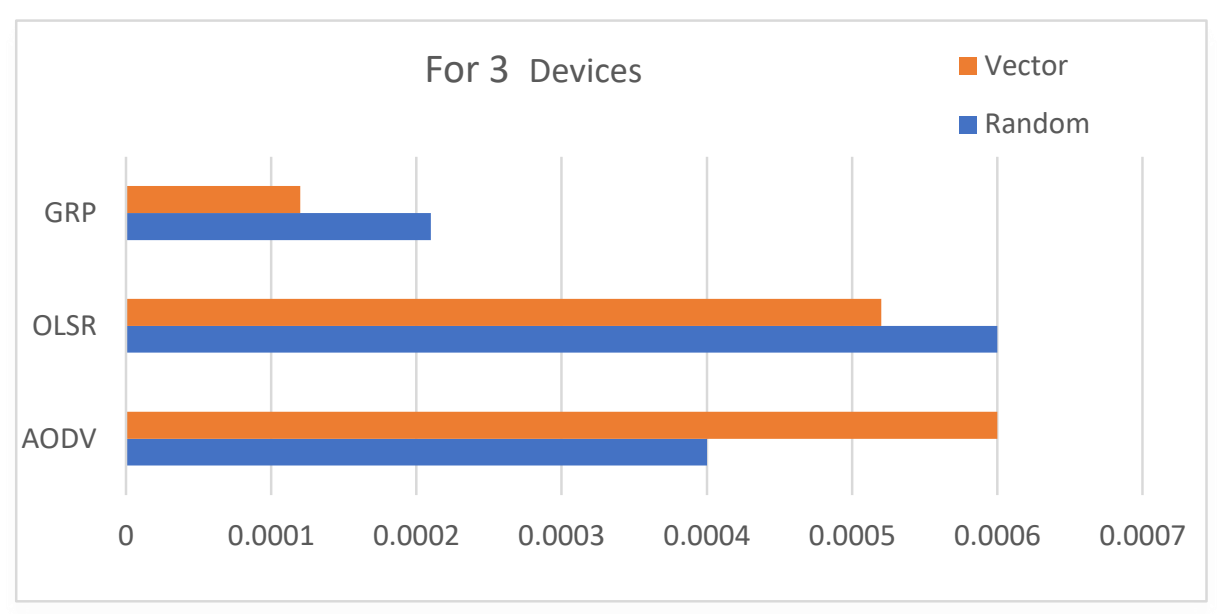

Fig.9. Delay (3 devices)

We have assessed the three execution measures with various portability models and HTTP as movement sort while taking 3 and 5 as the smart device thickness. The OLSR demonstrates the best execution as far as throughput, and end-to-end delay. 


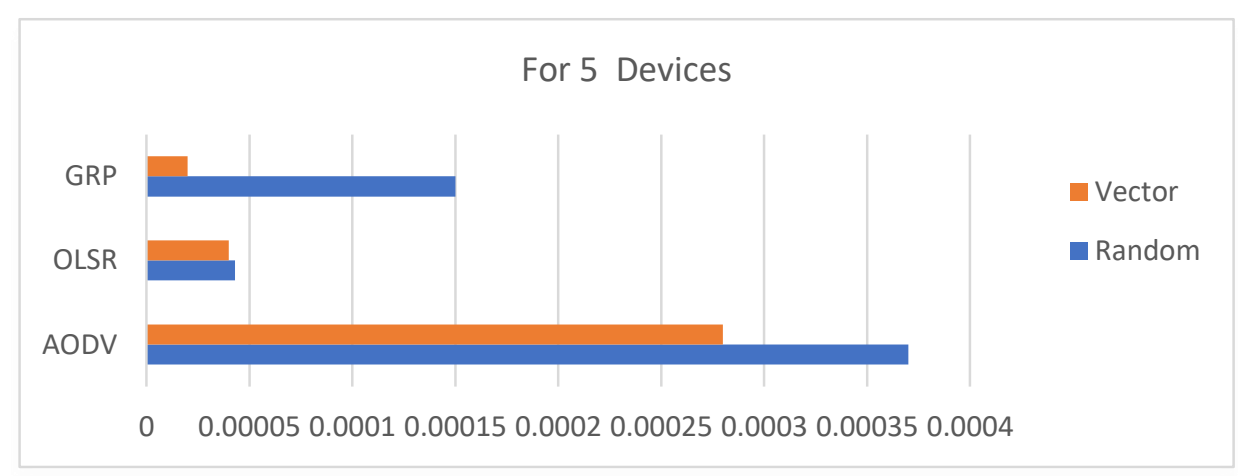

Fig.10. Delay (5 devices)

The proposed MANET in IoT is a collection of autonomous portable smart devices that can convey the information to one another through Wi-Fi waves. The link is created in the real time that makes the network completely dispersed and can work at wherever without the assistance of any access point. So that this property makes the network so strong. The MANET of smart devices works as infrastructure less network. Rather, smart devices themselves frame the system and convey through a method for remote correspondences.

\section{Conclusions}

After researching a lot of how MANET networks work in IoT and which are its advantages and disadvantages, I get to the conclusion that this kind of networks could help people in many situations, some of them in critical situations. But as far as doesn't support by itself the Ad-hoc mode it's not likely to think that some application could use this kind of networks for the general public. The MANET networks among smart devices in IoT is tested and implemented.

\section{References}

[1] Li, Shancang, Li Da Xu, and Shanshan Zhao. "The internet of things: a survey." Information Systems Frontiers 17.2 (2015): 243-259.

[2] Alam, Tanweer. "Middleware Implementation in Cloud-MANET Mobility Model for Internet of Smart Devices", International Journal of Computer Science and Network Security, 17(5), 2017. Pp. 86-94.

[3] Bellavista, Paolo, Giuseppe Cardone, Antonio Corradi, and Luca Foschini. "Convergence of MANET and WSN in IoT urban scenarios." IEEE Sensors Journal 13, no. 10 (2013): 3558-3567.

[4] Alam, Tanweer, and Mohammed Aljohani. "Design and implementation of an Ad Hoc Network among Android smart devices." In Green Computing and Internet of Things (ICGCIoT), 2015 International Conference on, pp. 1322-1327. IEEE, 2015. DOI: https://doi.org/10.1109/ICGCIoT.2015.7380671

[5] Sharma, Abhilash, Tanweer Alam, and Dimpi Srivastava. "Ad Hoc Network Architecture Based on Mobile IPV6 Development." Advances in Computer Vision and Information Technology 224 (2008).

[6] Alam, Tanweer, and Mohammed Aljohani. "An approach to secure communication in mobile ad-hoc networks of Android devices." In 2015 International Conference on Intelligent Informatics and Biomedical Sciences (ICIIBMS), pp. 371-375. IEEE, 2015. DOI: https://doi.org/10.1109/iciibms.2015.7439466 
[7] Aljohani, Mohammed, and Tanweer Alam. "An algorithm for accessing traffic database using wireless technologies." In Computational Intelligence and Computing Research (ICCIC), 2015 IEEE International Conference on, pp. 1-4. IEEE, 2015. DOI: https://doi.org/10.1109/iccic.2015.7435818

[8] Alam, Tanweer, and Mohammed Aljohani. "Design a new middleware for communication in ad hoc network of android smart devices." In Proceedings of the Second International Conference on Information and Communication Technology for Competitive Strategies, p. 38. ACM, 2016. DOI: https://doi.org/10.1145/2905055.2905244

[9] Alam T, Benaida M. The Role of Cloud-MANET Framework in the Internet of Things (IoT). International Journal of Online Engineering (iJOE). 2018;14(12):97-111. DOI: https://doi.org/10.3991/ijoe.v14i12.8338

[10] Alam, Tanweer. (2018) "A reliable framework for communication in internet of smart devices using IEEE 802.15.4." ARPN Journal of Engineering and Applied Sciences 13(10), 3378-3387.

[11] Alam T, Benaida M. CICS: Cloud-Internet Communication Security Framework for the Internet of Smart Devices. International Journal of Interactive Mobile Technologies (iJIM). 2018 Nov 1;12(6):74-84. DOI: https://doi.org/10.3991/ijim.v12i6.6776

[12] Bruzgiene, Rasa, Lina Narbutaite, and Tomas Adomkus. "MANET Network in Internet of Things System." In Ad Hoc Networks. InTech, 2017.

[13] Alameri, I. A. "MANETS and internet of things: the development of a data routing algorithm." Engineering, Technology \& Applied Science Research 8, no. 1 (2018): 2604-2608.

[14] Alam, Tanweer. "Fuzzy control based mobility framework for evaluating mobility models in MANET of smart devices." ARPN Journal of Engineering and Applied Sciences 12, no. 15 (2017): 4526-4538.

[15] Noorul, Thebiga M., R. Suji Pramila, and Noorul Islam. "An analysis of routing protocols in MANETs and Internet of things." In IoT and Application (ICIOT), 2017 International Conference on, pp. 1-8. IEEE, 2017.

[16] Tanweer Alam, "Blockchain and its Role in the Internet of Things (IoT)", International Journal of Scientific Research in Computer Science, Engineering and Information Technology, pp. 151-157, 2019. DOI: https://doi.org/10.32628/CSEIT195137

[17] Yu, Sui, Lichen Zhang, Lixia Li, Bin Yan, Zhipeng Cai, and Lizong Zhang. "An Efficient Interest-aware Data Dissemination Approach in Opportunistic Networks." Procedia Computer Science 147 (2019): 394399.

[18] Karlsson, Jonny, Laurence S. Dooley, and Goran P. Pulkkis. "Secure Routing for MANET Connected Internet of Things Systems." (2018).

[19] Kachouie, N.N., Fieguth, P., Ramunas, J. and Jervis, E., 2006. Probabilistic model-based cell tracking. International Journal of Biomedical Imaging, 2006.

[20] Moe, Marie EG, Bjarne E. Helvik, and Svein J. Knapskog. "TSR: Trust-based secure MANET routing using HMMs." In Proceedings of the 4th ACM symposium on QoS and security for wireless and mobile networks, pp. 83-90. ACM, 2008.

[21] Li L., Xu X., Cai Y. (2006) Gradient-Based Autoconfiguration for Hybrid Mobile Ad Hoc Networks. In: Gerndt M., Kranzlmüller D. (eds) High Performance Computing and Communications. HPCC 2006. Lecture Notes in Computer Science, vol 4208. Springer, Berlin, Heidelberg.

\section{Authors' Profiles}

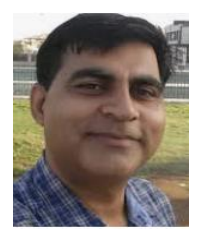

Tanweer Alam is with the Department of computer science, the Islamic University of Madinah since 2013. He is awarded by Ph.D. (Computer Science and Engineering), M.Phil. (Computer Science), MTech (Information Technology), MCA (Computer Applications) and M.Sc. (mathematics). His area of research including Mobile Ad Hoc Network (MANET), Internet of Things, Cloud Computing and wireless networking. He is a single author of twelve computer science textbooks. He is the member of various associations such as International Association of 
Computer Science and Information Technology (IACSIT), International Association of Engineers, Internet Society (ISOC), Computer Science Teachers Association (CSTA), Indian Society of Technical Education (ISTE) etc..

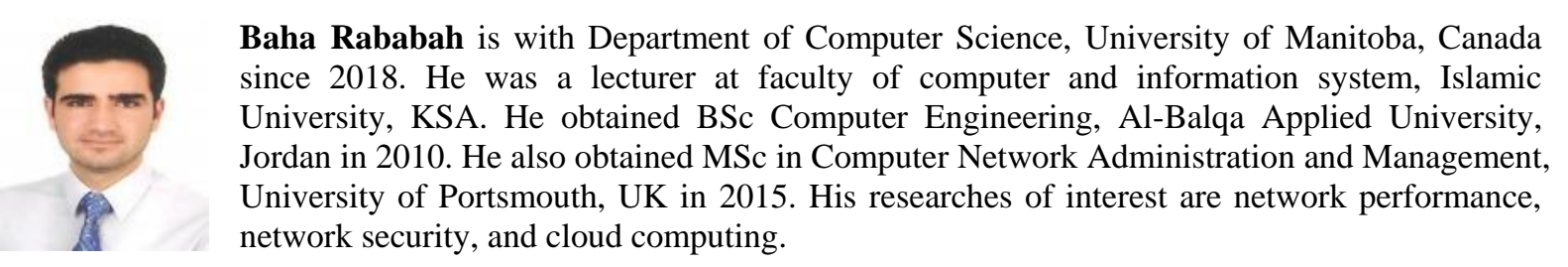

Baha Rababah is with Department of Computer Science, University of Manitoba, Canada since 2018. He was a lecturer at faculty of computer and information system, Islamic University, KSA. He obtained BSc Computer Engineering, Al-Balqa Applied University, Jordan in 2010. He also obtained MSc in Computer Network Administration and Management, network security, and cloud computing.

How to cite this paper: Tanweer Alam, Baha Rababah, "Convergence of MANET in Communication among Smart Devices in IoT", International Journal of Wireless and Microwave Technologies(IJWMT), Vol.9, No.2, pp. 1-10, 2019.DOI: 10.5815/ijwmt.2019.02.01 